\title{
North American Perpendicular Magnetic Recording Conference Highlighted Implementation of Technology
}

The first North American Perpendicular Magnetic Recording Conference (NAPMRC) debuted in Coral Gables, Florida, on January 7-9, 2002, organized and co-chaired by Sakhrat Khizroev and Dmitri Litvinov of Seagate Research (Pittsburgh, Pa.). The University of Miami in Coral Gables was the principal sponsor. The conference provided a review of the latest progress in the development of perpendicular magnetic recording as well as a discussion of the advantages, challenges, and timing of the transition to the technology. Planned as a highly selective meeting to attract key representatives of the data storage industry and academia, the conference assembled over 100 delegates representing more than 40 key industrial and academic organizations involved in magnetic data storage from the United States, Japan, the United Kingdom, South Korea, Switzerland, and France.

The conference convened during a time that is critical to future advances in data storage technologies. As conventional recording schemes rapidly approach the fundamental (superparamagnetic) limit in areal bit density, beyond which the recording data become unstable, it is believed that the perpendicular magnetic recording approach will allow researchers to sustain the current rate of technological advances for the next several generations of mass storage solutions.

NAPMRC had a single-session format with 34 invited talks focusing on six major topics pertinent to the successful implementation of the technology: Perpendicular Magnetic Recording Media, Perpendicular Magnetic Recording Heads, Theory of Perpendicular Recording, Measurement and Characterization, Channels for Perpendicular Recording, and Perpendicular Recording Systems and System Integra- tion. A concluding session was devoted to a discussion of the future of magnetic data storage, emphasizing the role of perpendicular recording. A number of contributed papers were presented at the special poster session.

As portrayed by Jack Judy, director of the Center for Micromagnetics and Information Technologies at the University of Minnesota, the conference resonated on the background of recent developments in the data storage industry. Among the highlights of the NAPMRC was an overview of the history of perpendicular recording given by Shun-ichi Iwasaki of the Tohoku Institute of Technology, Japan. Iwasaki is widely accepted as the inventor of perpendicular magnetic recording. Steven Lambert of Maxtor Corp. presented an overview of a fully functional prototype of a 31-Gbit/in. ${ }^{2}$ hard drive based on perpendicular recording developed by Maxtor. The Maxtor team ran a server operating system on the developed hard drive for one month. A number of presentations (by Judy, Naoki Honda of AIT, Masaaki Futamoto of CRL Hitachi, Hiroaki Muraoka of Tohoku University, Mason Williams and Roger Wood of IBM, and Mike Mallary of Maxtor) were devoted to design guidelines and future prospects for perpendicular recording. The consensus was that the areal densities of up to $\sim 1$ Tbit/in. ${ }^{2}$ are likely to be realized using perpendicular magnetic recording. Mark Kryder of Seagate Research gave an overview of the future of magnetic data storage, including an account of the latest results in heat-assisted magnetic recording (HAMR) and magnetic recording based on patterned media. It was suggested that future technologies such as HAMR or recording on patterned media would be based on perpendicular recording, owing to its advantageous properties with respect to extremely high areal bit densities, thus making perpendicular recording not merely a temporary solution, but rather a necessary basis for a number of future generations of magnetic data storage devices.

The invited papers will appear in the conference proceedings, which will be published in a July 2002 issue of IEEE Transactions on Magnetics.

The conference was co-sponsored/ endorsed by the IEEE Magnetics Society, the National Storage Industry Consortium, and the Materials Research Society, and was endorsed by leading academic centers involved in magnetic data storage: the Center for Micromagnetics and Information Technologies (MINT) of the University of Minnesota, the Center for Materials for Information Technology (MINT) of the University of Alabama, the Center for Magnetic Recording Research (CMRR) of the University of CaliforniaSan Diego, the Center for Research on Information Storage Materials (CRISM) of Stanford University, and the Data Storage Systems Center (DSSC) of Carnegie Mellon University. Veeco Instruments Inc. provided partial financial support for the conference banquet.

Local organizational support was provided by the faculty of the Department of Physics of the University of Miami, represented by Joshua Cohn and Josef Ashkenazi. The honorary co-chairs of the conference were Iwasaki Tohoku Institute and Stanley Charap of Carnegie Mellon University.

The next NAPMRC is tentatively scheduled for January 6-8, 2003. More information about NAPMRC can be found at the conference Web site at http:/ / www.napmrc.org.
August 1-2, 2002

International Workshop on

Processing and Applications of Superconductors

Park Vista Resort Hotel

Gatlinburg, Tennessee, USA

See page 369 for details.
October 23-25, 2002

2nd International Workshop on Zinc Oxide

Holiday Inn

Dayton, Ohio
December 2-6, 2002

2002 MRS Fall Meeting

Hynes Convention Center

and Sheraton Boston Hotel

Boston, MA

See page 380 for details.

For the most current information on these and future events, visit www.mrs.org/meetings/ 